Supporting Information

\title{
Nonvolatile negative optoelectronic memory based on ferroelectric thin films
}

Nan Yang, ${ }^{\dagger}$ Chuan-Zhu Hu, ${ }^{\dagger}$ Zhong-Qi Ren, ${ }^{\dagger}$ Si-Yao Bao, ${ }^{\dagger}$ Bo-Bo Tian, ${ }^{\dagger}$ Fang-Yu Yue,,$^{\dagger}$ Ping-Hua Xiang, ${ }^{\dagger, \dagger}$ Ni Zhong, ${ }^{*}, \dagger,+$ Chun-Gang Duan, ${ }^{\dagger}, \dot{\dagger}$ and Jun-Hao Chu ${ }^{\dagger,+}$

${ }^{\dagger}$ Key Laboratory of Polar Materials and Devices (MOE), Department of Electronics, East China Normal University, Shanghai 200241, China

¥ Collaborative Innovation Center of Extreme Optics, Shanxi University, Shanxi 030006, China

* Author to whom correspondence should be addressed: nzhong@ee.ecnu.edu.cn 

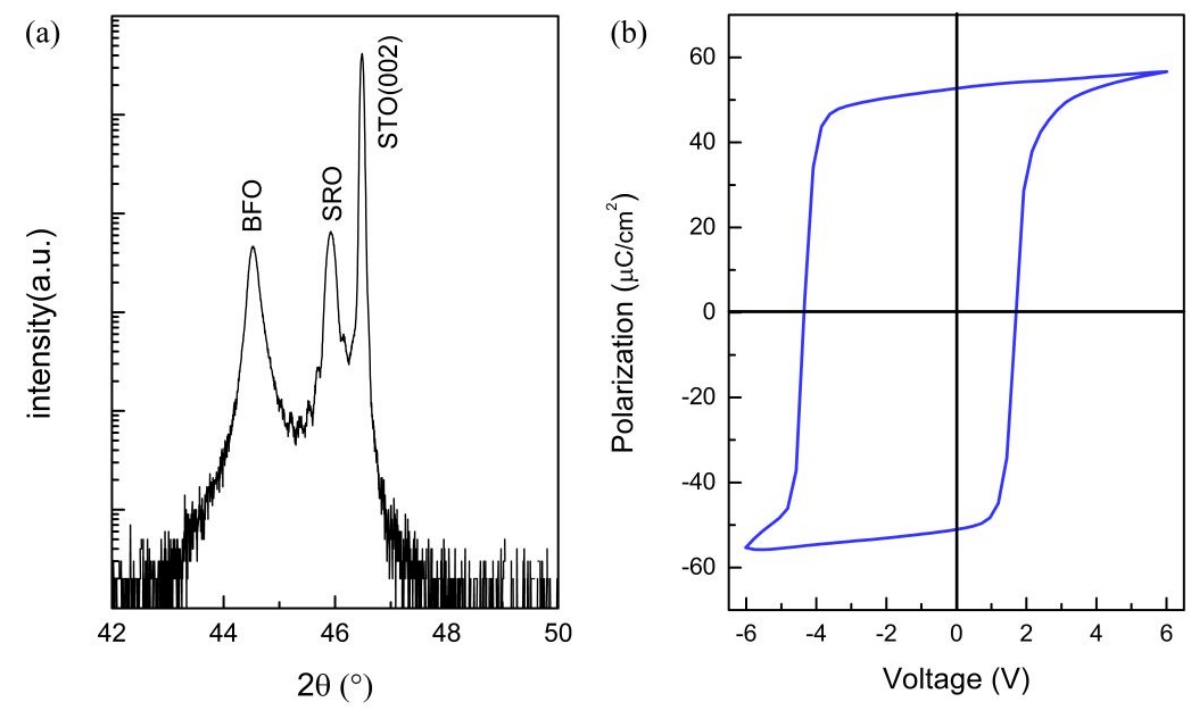

Figure S1. (a) XRD $\theta-2 \theta$ scans for the BFO thin film grown on SRO buffered STO substrate. (b) Polarization-electric field (P-E) hysteresis loops at $100 \mathrm{~K}$ of the BFO thin film. The XRD result indicated good epitaxy of BFO/SRO films and we did not observe any reflections that would be indicative of second phases. In P-E loop, the value $2 \mathrm{Pr}$ is higher than $100 \mu \mathrm{C} / \mathrm{cm}^{2}$, suggesting the excellent ferroelectric characterization of our BFO films. The asymmetricity of P-E loop can be attributed to the asymmetric top and bottom electrodes. 

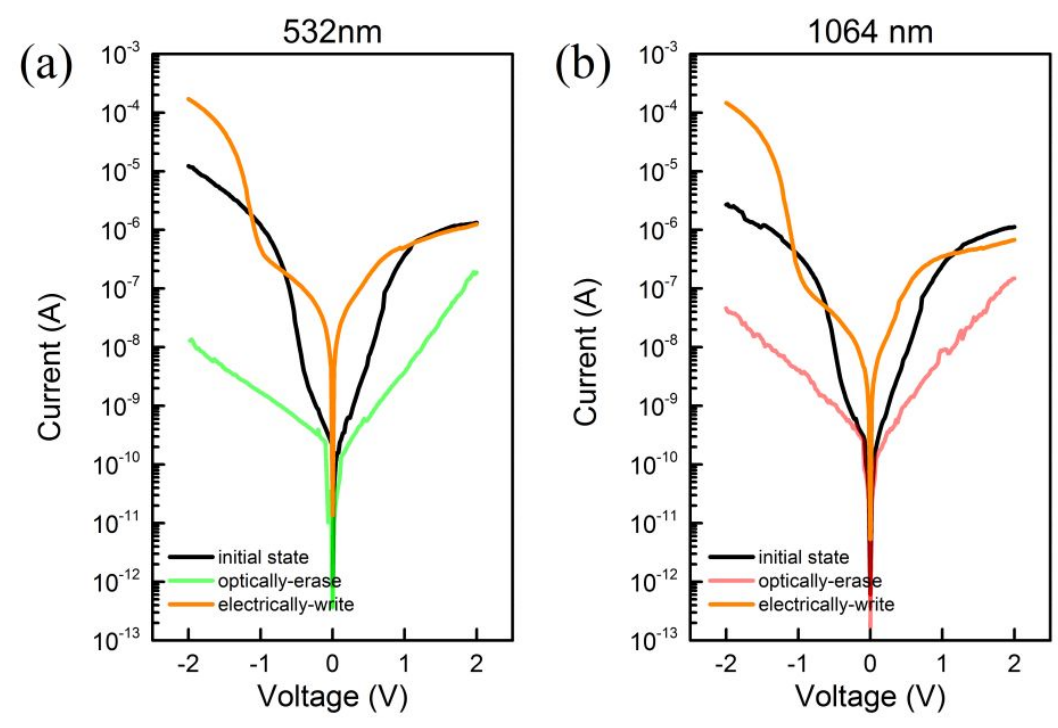

Figure S2. NPC effect induced from (a) 532 and (b) $1064 \mathrm{~nm}$ laser illumination. Illumination was performed in the ITO/BFO/SRO devices by green $(532 \mathrm{~nm}, 2.33 \mathrm{eV})$ and NIR $(1064 \mathrm{~nm}, 1.17 \mathrm{eV})$ laser, respectively. The APC effect was produced in over a broadband absorption that is much smaller than the band gap of the BFO film (2.7 $\mathrm{eV})$. 


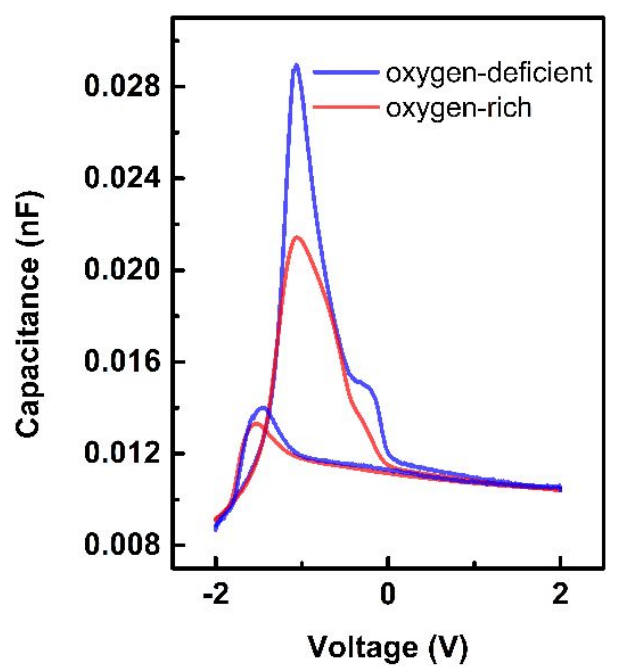

Figure S3. $C$ - $V$ curves measured at $1 \mathrm{MHz}$ after annealing treatment in an oxygen-rich and oxygen-deficient environment, respectively. Firstly, the device was annealed in the oxygen-deficient environment, and it is found that the intensity of the two peaks range from -1 to $0 \mathrm{~V}$ exhibit a clear enhancement. Moreover, the following oxygen-rich annealing treatment results in the suppression of the peaks and the capacitance peaks caused by oxygen vacancies almost disappear. This result strongly indicates that the effect of annealing treatment on oxygen vacancy in our devices. 


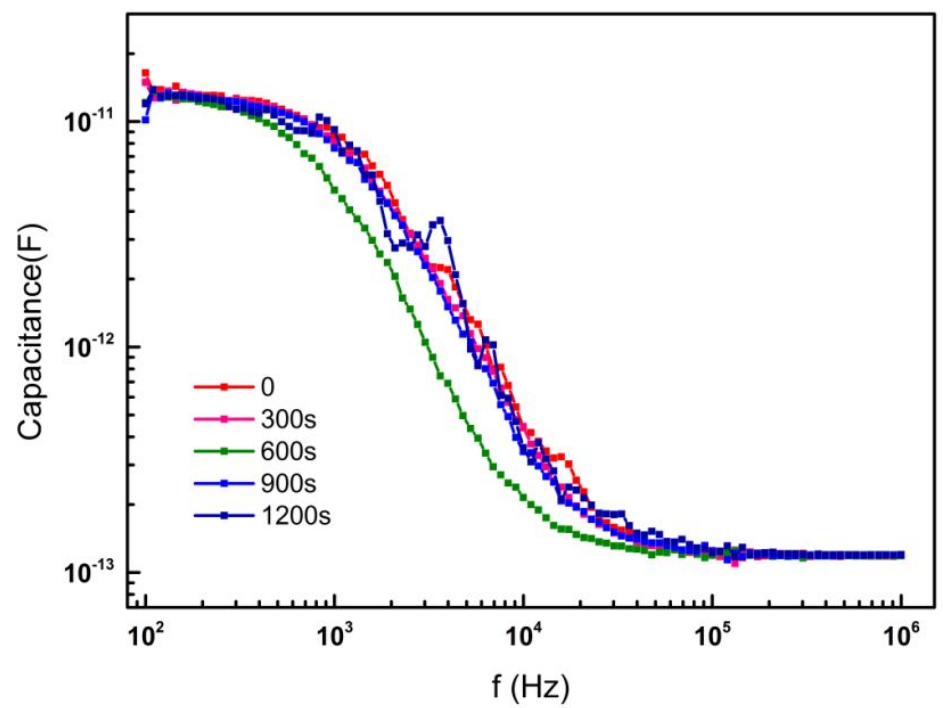

Figure S4. Retention performance of the $C-f$ curves after laser illumination for $1200 \mathrm{~s}$. The $C-f$ curves remain stable in air after $10^{3} \mathrm{~s}$ after removing the illumination source, therefore allowing the nonvolatile storage of the capacitance information to be written optically. 


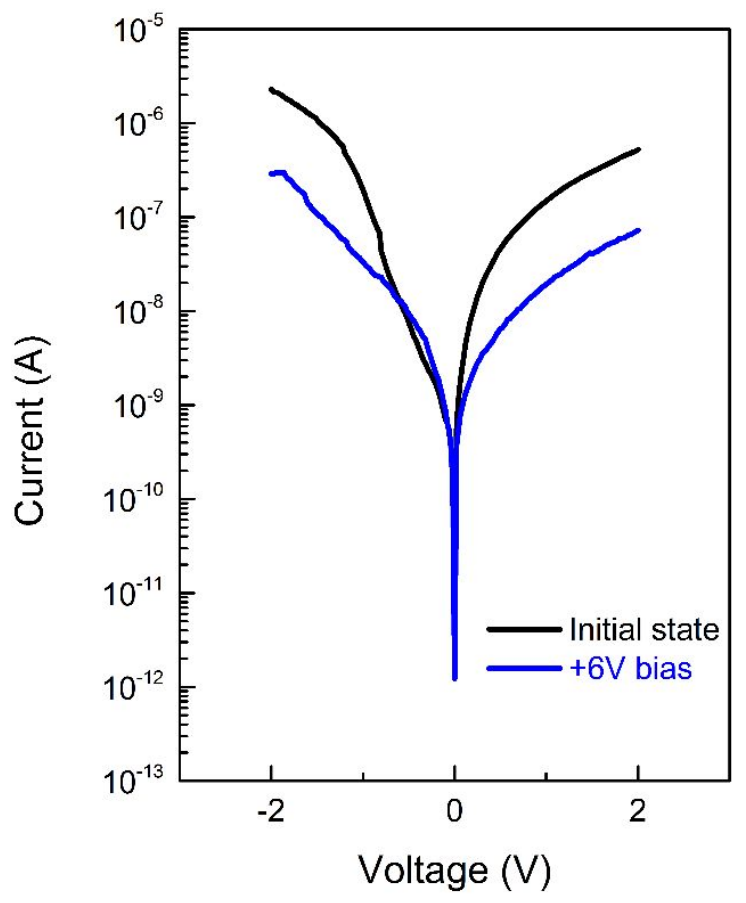

Figure S5. $I V$ curves obtained in dark (black) and after a voltage pulse of $6 \mathrm{~V}$ for 500 $\mu$ s (blue). The reason consistent with the results of light illumination is that the electrons trapped in the oxygen vacancies of the interfacial region will be released under high positive voltage. 